\title{
Proteomic analysis of murine Piwi proteins reveals a role for arginine methylation in specifying interaction with Tudor family members
}

\author{
Vasily V. Vagin, ${ }^{1}$ James Wohlschlegel, ${ }^{2}$ Jun $\mathrm{Qu},{ }^{3}$ Zophonias Jonsson, ${ }^{2}$ Xinhua Huang, ${ }^{2}$ \\ Shinichiro Chuma, ${ }^{4}$ Angelique Girard, ${ }^{1}$ Ravi Sachidanandam, ${ }^{5}$ Gregory J. Hannon, ${ }^{1,6}$ \\ and Alexei A. Aravin ${ }^{1,7}$ \\ ${ }^{1}$ Watson School of Biological Sciences, Howard Hughes Medical Institute, Cold Spring Harbor Laboratory, Cold Spring Harbor, \\ New York 11724, USA; ${ }^{2}$ Department of Biological Chemistry, University of California at Los Angeles, Los Angeles, California \\ 90095, USA; ${ }^{3}$ Department of Pharmaceutical Sciences, State University of New York at Buffalo and New York State Center of \\ Excellence in Bioinformatics and Life Sciences, Buffalo, New York 14203, USA; ${ }^{4}$ Department of Development and \\ Differentiation, Institute for Frontier Medical Sciences, Kyoto University, Shogoin, Sakyo-ku, Kyoto 606-8507 Japan; \\ ${ }^{5}$ Department of Genetics and Genomic Sciences, Mount Sinai School of Medicine, New York, New York 10029, USA
}

\begin{abstract}
In germ cells, Piwi proteins interact with a specific class of small noncoding RNAs, piwi-interacting RNAs (piRNAs). Together, these form a pathway that represses transposable elements, thus safeguarding germ cell genomes. Basic models describe the overall operation of piRNA pathways. However, the protein compositions of Piwi complexes, the critical protein-protein interactions that drive small RNA production and target recognition, and the precise molecular consequences of conserved localization to germline structures, call nuage, remains poorly understood. We purified the three murine Piwi family proteins, MILI, MIWI, and MIWI2, from mouse germ cells and characterized their interacting protein partners. Piwi proteins were found in complex with PRMT5/ WDR77, an enzyme that dimethylates arginine residues. By immunoprecipitation with specific antibodies and by mass spectrometry, we found that Piwi proteins are arginine methylated at conserved positions in their $\mathbf{N}$ termini. These modifications are essential to direct complex formation with specific members of the Tudor protein family. Recognition of methylarginine marks by Tudor proteins can drive the localization of Piwi proteins to cytoplasmic foci in an artificial setting, supporting a role for this interaction in Piwi localization to nuage, a characteristic that correlates with proper operation of the piRNA pathway and transposon silencing in multiple organisms.
\end{abstract}

[Keywords: Arginine methyation; piRNAs; transposon silencing; tudor proteins]

Supplemental material is available at http://www.genesdev.org.

Received April 24, 2009; revised version accepted June 8. 2009.

Argonaute (Ago) proteins bind small RNAs to form the core of the RNA-induced silencing complex (RISC), which is responsible for both artificial RNAi and natural regulation of gene expression by endogenous small RNAs. In all Metazoa, the Argonaute family contains multiple members, which can be placed into the so-called Ago and Piwi clades, based on their sequence conservation.

Members of the Ago clade are found in all cell types. In contrast, Piwi proteins are a signature feature of germ cells in multicellular animals. Their associated piwi-

Corresponding authors.

${ }^{6}$ E-MAIL hannon@cshl.edu; FAX (516) 367-8874.

${ }^{7}$ E-MAIL aravin@cshl.edu; FAX (516) 367-8874.

Article published online ahead of print. Article and publication date are online at http://www.genesdev.org/cgi/doi/10.1101/gad.1814809. Freely available online through the Genes \& Development Open Access option. interacting RNA (piRNA) populations have been characterized from Caenorhabditis elegans, Drosophila, zebrafish, Xenopus, and mammals (Aravin et al. 2006; Girard et al. 2006; Saito et al. 2006; Vagin et al. 2006; Brennecke et al. 2007; Batista et al. 2008; Das et al. 2008; Houwing et al. 2008; Wilczynska et al. 2009), and this has pointed to conserved roles in the suppression of mobile genetic elements (Aravin et al. 2007a). Post-transcriptional mechanisms clearly contribute to piRNA-directed silencing, since small RNA-guided cleavage inactivates transposon mRNAs. However, in mice, two Piwi family members, MILI and MIWI2, also specify de novo DNA methylation of transposon sequences in embryonic germ cells, leading to their transcriptional repression (Aravin et al. 2007b; Carmell et al. 2007; Kuramochi-Miyagawa et al. 2008).

The biogenesis mechanisms that generate piRNAs differ from those that produce other small RNA classes 
(Aravin et al. 2007a). MicroRNAs (miRNAs) and siRNAs emerge from double-stranded precursors through the action of conserved RNaseIII family members, including Dicer and Drosha, whereas Dicer is dispensable for piRNA generation in Drosophila and zebrafish (Vagin et al. 2006; Houwing et al. 2007). Instead, piRNAs are produced from genomic loci, piRNA clusters that give rise to long, single-stranded precursor transcripts. These appear to produce piRNAs through two, likely distinct, biochemical pathways (Brennecke et al. 2007). Primary piRNA biogenesis involves direct sampling of precursor transcripts by an unknown machinery. Precursor transcripts from piRNA clusters can also participate, along with transposon mRNAs, in a loop, called the ping-pong cycle, in which piRNA-directed cleavage of each long RNA by Piwi proteins produces the $5^{\prime}$ ends of new piRNAs (Brennecke et al. 2007; Gunawardane et al. 2007). This results in a mixture of sense and antisense species that tend to be enriched for sequences corresponding to transposons that are actively expressed (Brennecke et al. 2008).

Overall, the domain structures of Piwi and Ago proteins are highly similar; both have PAZ domains, which engage the 3' ends of small RNAs (Hutvagner and Simard 2008), an RNase H-like motif in the Piwi domain that catalyzes small RNA-directed target cleavage, and a binding pocked in the mid-domain that holds the $5^{\prime}$ end of the small RNA guide. However, the proteins that interact with Ago and Piwi family members differ substantially.

Biochemical purification of Ago-RISCs has identified many components whose roles in small RNA pathways or whose interactions with these effector complexes are conserved between Drosophila and mammals. Among these are Dicer and its dsRBD-containing cofactors (TRBP, R2D2, Loqs), the putative RNA helicase, MOV10/Armitage, components of P-bodies, including GW182/TNRC6B, and a nuclear transport receptor, Importin-8 (Chendrimada et al. 2005; Gregory et al. 2005; Liu et al. 2005; Meister et al. 2005; Weinmann et al. 2009).

The composition of Piwi complexes has been investigated less extensively. Thus far, interactions have been reported in flies with a chromatin-associated protein, HP1 , and in rodents with a nuclease, RecQ1, and a kinesin motor protein, KIF17b (Kotaja et al. 2006; Lau et al. 2006; Brower-Toland et al. 2007). Here, we report the purification and analysis of complexes containing each of the three mouse Piwi proteins, MILI, MIWI, and MIWI2, from germ cells. Among the proteins that interact with these family members were an arginine methyltransferase, PRMT5, and its cofactor, WDR77/MEP50 (Friesen et al. 2002). The three mouse Piwi proteins, as well as family members from other organisms, contain multiple RG/RA sites at their $\mathrm{N}$ termini that serve as potential methylation sites, and arginine methylation of Piwi proteins was confirmed by mass spectrometry and antibody recognition. These arginine methyl marks are read by a family of Tudor domain proteins, whose individual members show specificity for interaction with MIWI, MILI, or MIWI2. Tudor family proteins show dynamic patterns of expression during spermatogenesis, and some colocalize with specific Piwi family proteins in cytoplasmic nuage. Anal- ysis of mutant animals supports critical roles for Tudor proteins, likely through their interactions with Piwi family members, in the piRNA pathway.

\section{Results}

\section{Purification of Piwi complexes from germ cells}

To facilitate purification of Piwi RNP complexes, we established transgenic mouse lines expressing epitopetagged MILI, MIWI, and MIWI2. In each case, two independent transgenes were constructed by fusing either $3 \mathrm{xmyc}$ or $3 \mathrm{xFlag} / \mathrm{HA}$ tags at the $\mathrm{N}$ terminus of each target protein via BAC recombineering (Ohtsuka et al. 2009). Following pronuclear injection, these constructs produced animals in which the modified protein was expressed under its own, endogenous promoter. Several independent founder lines were established for each construct. All gave the expected product on Western blots and reproduced the developmentally timed expression pattern and subcellular localization that had been documented for the corresponding endogenous protein (data not shown).

In males, MIWI2 expression begins in developing germ cells during late embryogenesis (approximately embryonic day 14.5 [E14.5]) and persists until shortly after birth. MILI expression begins earlier (approximately E12.5), and the protein is continuously present in germ cells until the haploid round spermatid stage. MIWI is not expressed until the pachytene stage of meiosis (approximately postnatal day 14 [P14]). Accordingly, we isolated MIWI2 and MILI from embryonic testes (E17.5) and MILI and MIWI complexes from adult animals $(\sim 3$ mo old). Complexes were recovered using either a single-step procedure with antibodies to endogenous proteins or a multistep purification that involved sequential binding to anti-Flag and anti-HA beads. After elution from beads, recovered proteins were analyzed by Multidimensional Protein Identification Technology (MudPIT). Confident calls of interacting proteins took advantage of comparisons of results obtained with multiple independent samples and two different tags. For reference, these were compared with identical purifications from nontransgenic animals, which are essentially null for the tagged protein. A compilation of the MIWI-, MILI-, and MIWI2-associated proteomes is given in Supplemental Table S1.

Overall, we failed to detect the many identified components of canonical Ago RISC in association with Piwi family members, including Dicer, TRBP, or GW182/ TNRC6B. Instead, we found a rich, but mostly distinct Piwi-associated proteome. For all three family members, we saw a general enrichment of proteins identified previously as components of germline granules, including chromatoid bodies, consistent with the demonstrated subcellular compartmentalization of Piwi proteins (see Supplemental Table S1).

We did not detect mouse RecQ1 or HP1 homologs, which were shown previously to interact with Piwi family members in other organisms (Lau et al. 2006; Brower-Toland et al. 2007). MIWI and MIWI2 immunocomplexes did 
contain a germline-specific paralog of Mov10, Mov10I1. Mov10I1 is a homolog of Armitage, a helicase required for the function of the piRNA pathway in Drosophila germ cells (Vagin et al. 2006). Its paralog, Mov10, has been reported to interact with Ago clade proteins (Meister et al. 2005), suggesting at least some commonality between Piwi and Ago RISCs.

With MIWI and MIWI2, we also detected complex formation with $\mathrm{MVH}$, the mouse homology of Vasa, a helicase that has long served as a marker of germ cells. Vasa is functionally linked to efficient operation of the piRNA pathway in Drosophila germ cells. MVH colocalizes with MIWI in chromatoid bodies in meiotic spermatocytes and with MILI in both chromatoid bodies and smaller cytoplasmic RNP granules present in prospermatogonia, spermatogonia, and spermatocytes (data not shown). Complex formation was not observed between MILI and MVH, supporting the specificity of the MVH-MIWI and MVHMIWI2 complexes.

Piwi proteins associate with and are modified by an arginine-methylase complex

Common components of all Piwi complexes, irrespective of developmental stage, were the arginine methyltransferase, PRMT5, and its cofactor, WDR77 (Fig. 1A). After dual Flag/HA purification of MIWI, PRMT5 and WDR77 were represented by 20 and 14 peptides, corresponding to $21.6 \%$ and $18.4 \%$ protein coverage, respectively. This interaction could be confirmed by Western blotting and was insensitive to RNase treatment (Fig. 1B). Similar interactions were detected with MILI and MIWI2 (Fig. 1B; data not shown).

To validate the observed interactions and to test the degree to which they were dependent on the ongoing operation of the piRNA pathway, we used 293 cells, which neither mount a piRNA response nor load ectopically expressed Piwi proteins with small RNAs (data not shown). Myc-tagged MILI, MIWI, or MIWI2 were coexpressed with Flag/HA-tagged PRMT5 or WDR77. Interaction was tested by probing anti-myc immunoprecipitates with anti-HA antibodies. None of the Piwi proteins appeared to interact appreciably with PRMT5. However, all three showed binding to WDR77 (Fig. 1C), which might bridge interactions between Piwis and PRMT5.

PRMT5 and WDR77 form a methylosome complex that symmetrically dimethylates arginine and lysine residues in many proteins, including histones $\mathrm{H} 3$ and $\mathrm{H} 4$, spliceosomal proteins Sm D1 and Sm D3, and the p53 tumor suppressor (Pal et al. 2004; Jansson et al. 2008). Since Piwis associated with the PRMT5/WDR77 complex, we examined the possibility that they, themselves, might serve as methylosome substrates. Flag/HA-tagged MIWI, immunoprecipitated from testis extracts, was recognized by an antibody (SYM10) that binds multiple symmetrically methylated arginine residues (Fig. 2A). MIWI could also be immunoprecipitated using the antimethylarginine antibody.

Costaining of adult testis sections using SYM10 and anti-MIWI antibodies produced strong signals in the

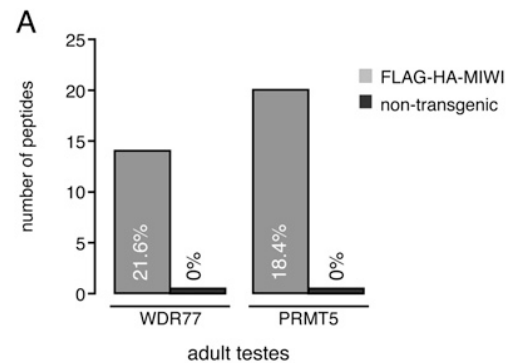

B
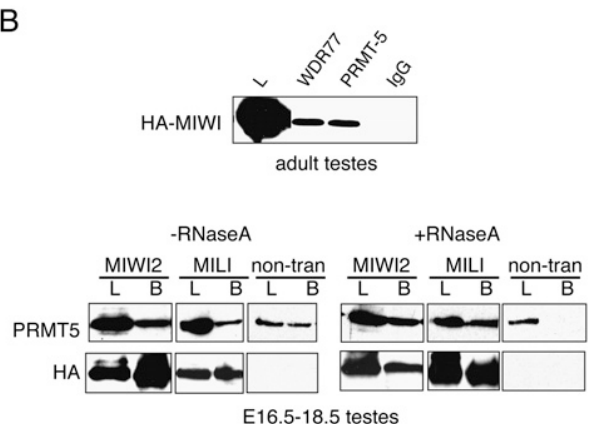

C

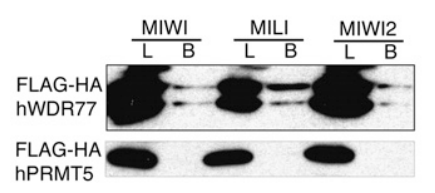

Figure 1. PRMT5/WDR77 complex associate with mouse Piwi proteins. (A) Presented are MudPIT analyses of immuoprecipitated 3xFlag-HA-MIWI complexes with the number of peptides and coverage (percent shown in each column) shown for HA elution. (B, top panel) PRMT5 or WDR77 was immunoprecipitated from adult testis extracts and probed on a Western blot with HA antibodies to detect Flag-HA-MIWI. (Bottom panel) Flag-HA-tagged MIWI2 or MILI was immunoprecipitated from embryonic testes (E16.5-E18.5) using Flag beads and probed with PRMT5 or HA antibodies. (L) Lysate; (B) bound fractions. The bands seen in immunoprecipitates from nontransgenic animals represent a nonspecific interaction between PRMT5 and the anti-Flag beads. (C) Tagged MIWI, MIWI2, or MILI proteins were immunoprecipitated from 293 cell extracts using myc beads and probed on Westerns with HA antibodies.

chromatoid bodies of round spermatids (Fig. 2B). Staining was also seen with ASYM10, an antibody, which recognizes asymmetrically methylated arginine residues, indicating an accumulation of proteins with both types of modifications in this cytoplasmic body (Fig. 2C). In spermatocytes at earlier stages of development and in embryonic gonocytes, we observed nuclear staining with SYM10 antibodies (Fig. 2C; Supplemental Fig. S1). Of course, many proteins bearing methyl marks are recognized by SYM10 and ASYM10. For example, Sm D1 and Sm D3 have been reported to shuttle between nuage and the nucleus in both mice and flies (Chuma et al. 2006). We therefore investigated which proteins contributed most prominently to the observed anti-methylarginine staining of chromatoid bodies. 
Vagin et al.

A
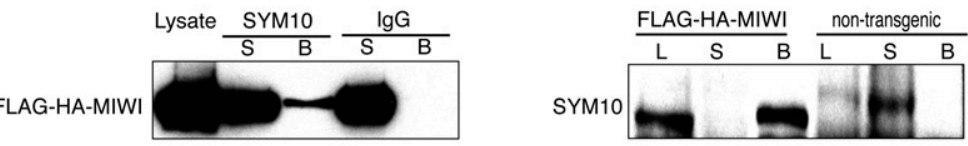

Figure 2. Arginine methylation of MIWI and the presence of arginine methylated proteins in the chromatoid body (CB). (A, left panel) Tagged MIWI protein was immunoprecipitaed from testes extracts with SYM10 (anti-symmetric dimethylarginine) and probed by Western with HA antibodies. (Right panel) Tagged MIWI was immunoprecipitated using Flag beads and tested on Western with SYM10 antibodies. (L) Lysate; (S) supernatant; (B) bound fractions. (B) Localization of symmetric dimethylarginine (SYM10) and MIWI was noted in the CB of round spermatids. Before the $\mathrm{CB}$ is formed, MIWI and Sym10 marks are not colocalized. In spermatocytes ( $\mathrm{Sp}$ ) MIWI is uniformly distributed in the cytoplasm, while Sym10 predominantly stain the nucleus. $(C)$ Localization of symmetric (SYM10) and asymmetric (ASYM14) arginine methylation marks was analyzed in testes of 20-d-old heterozygous and homozygous Miwi animals. (D) The PRMT5 partner, WDR77 localizes to CB in control animals $\left(\mathrm{Miwi}^{+/-}\right)$, but is lost in MIWI-deficient animals, while the $\mathrm{CB}$ markers, MVH and MILI, retain their localization in CBs. PRMT5 is uniformly distributed in the cytoplasm independently of MIWI.

B
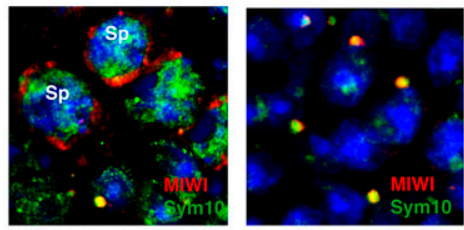

Miwi +/-
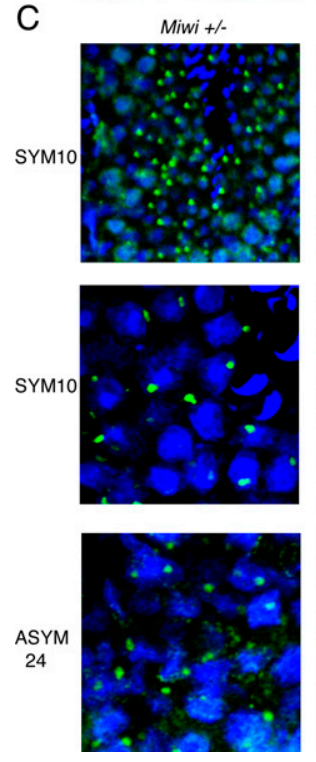

Miwi -/-
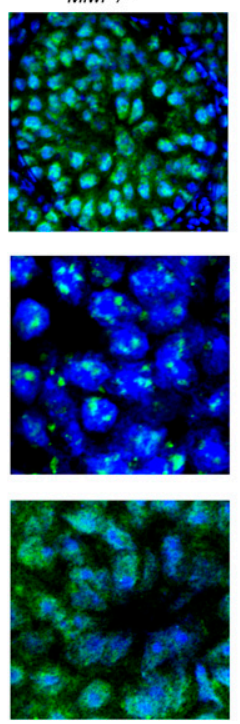

D
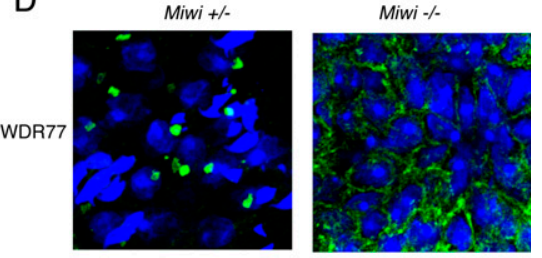

MVH
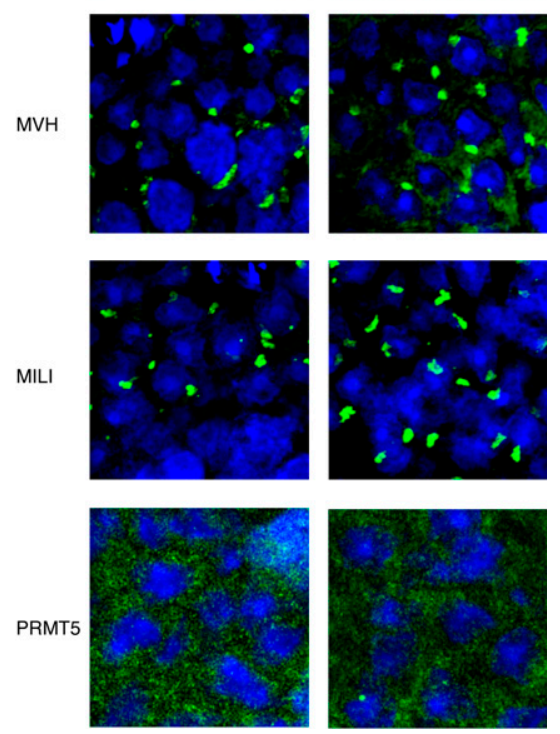

The round spermatids of Miwi-mutant mice $\left(\mathrm{Miwi}^{-/-}\right)$ (Fig. 2C) still contain chromatoid bodies, as indicated by the persistent localization of MVH, MILI, TDRD1, TDRD6, TDRD7, and TDRD9 in a prominent perinuclear granule (Fig. 2D; Supplemental Fig. S2). Yet, the staining of chromatoid bodies with anti-methylarginine antibodies was greatly reduced upon MIWI loss (Fig. 2C). In embryonic gonocytes WDR77 is uniformly distributed in the nucleus and cytoplasm (Supplemental Fig. S1), but it found prominently in the chromatoid bodies of round spermatids (Fig. 2D). WDR77 localization to chromatoid bodies is perturbed in Miwi mutants, while the uniform cytoplasmic localization of PRMT5 in the cytoplasm unaffected (Fig. 2D). Considered together, these data are consistent with a model in which MIWI is a major component of the chromatoid body that brings WDR77 to that structure through their residence in the same RNP complex.

\section{Sites of methylation in Piwi proteins}

We wished to determine the precise sites of arginine modification within mouse Piwi family proteins. This presents a significant challenge for conventional LC/MS/ MS due both to the intrinsically high charge states of methylarginine-containing peptides and to the intrinsic lability of methylarginine moieties, which precludes effective fragmentation of the peptide backbone ( $\mathrm{H}$ Wang et al. 2009). We therefore employed and extensively optimized a number of analytical advancements to achieve sufficient protein coverage of methylarginine-containing peptides and to permit localization of modified positions within those fragments.

MIWI and MILI were successfully purified from mouse testes using an anti-Flag monoclonal antibody. MIWI2 purifications did not yield sufficient material for analysis. These preparations were further purified and digested using a gel-free precipitation/on-pellet-digestion procedure (Duan et al. 2009). This provided higher peptide recovery and lower nonuniform peptide loss than gelbased preparation methods. Two proteolytic enzymes (trypsin and V8) and two fragmentation methods (CID and ETD/ were used separately for identification. Using two methods in parallel produced complementary proteolytic profiles, which was essential for improving both protein coverage and the identification of modified residues. For example, when a modified residue occurs in an arginine-rich domain, trypsin will generate very short, highly polar peptides that elude separation and sequencing, whereas V8 generates suitable peptide from such 
a domain. Although ETD is superior to CID for sequencing methylarginine peptides, the latter can provide unique fragmentation information that may reveal methylation symmetries. Samples were examined on a highresolution/accuracy MS analyzer (Orbitrap) and results were passed through a set of high stringency filtration criteria (see the Materials and Methods). All positions at which methylated arginine residues were called were cross-validated using multiple approaches.

We achieved $87 \%$ sequence coverage for MIWI and $83 \%$ for MILI (Supplemental Fig. S3). We were able to identify with high confidence three modified arginines on MIWI and six on MILI (Fig. 3A; Supplemental Fig. S4). Of the three modified sites in MIWI, two lay close to the N terminus at residues 14 and 49. Methylation may also occur on arginine residues nearer the $\mathrm{N}$ terminus, but definitive calls at these sites were prevented by low coverage in this area (Supplmental Fig. S3). R14 appeared in both monomethylated and symmetrically dimethylated forms. R49 and R371 were monomethylated. R14 and R49 are positioned in extended stretches of RG/RA sequences that are similar to mapped sites of PRMT5catalyzed methylation in other targets, such as Sm D1 and D3 and coilin (Fig. 3B, inset). In MILI, R74 was both monomethylated and symmetrically dimethylated. R100, R163, and R549 were symmetrically dimethylated and R95 was monomethylated. The majority of methylarginine residues in MILI are conserved in its orthologs from other mammalian species. R549 is located in an RWG motif between the PAZ and PIWI domains within the mid domain. Previous studies have not reported symmetric dimethylation in RWG motifs, but the analysis of this residue by multiple independent methods provides high confidence for the existence of this modification.

Potential sites for arginine methylation are a conserved feature of Piwi family proteins. Although sites in MIWI2 could not be mapped experimentally, it does contain multiple tandem RG/RA motifs near its $\mathrm{N}$ terminus. A similar configuration is seen in other vertebrates (humans, Xenopus, and zebrafish) as well as in invertebrates including Drosophila (Aub, Piwi, and AGO3), C. elegans (Prg-1), sea urchin (seawi), planaria (smedwi), and hydra (cniwi) (Fig. 3B; data not shown). There is an overall lack of conservation in $\mathrm{N}$-terminal regions among Piwi family proteins. Nevertheless, of all those available for examination, only $\operatorname{Prg}-2$, which has a foreshortened $\mathrm{N}$ terminus, lacks the motif. Interestingly, in contrast to prg-1 mutants, loss of prg-2 does not affect piRNA expression or germline development (Batista et al. 2008). Although the Piwi and Ago clades share overall protein structure, analogous RG/RA motifs are missing from human and mouse Ago1-4 and from Drosophila Ago1 and Ago2. Considered together, these observations indicate that $\mathrm{N}$-terminal arginine methylation may be a conserved feature that distinguishes RISCs containing Piwi family proteins from those formed around the Ago clade.

A
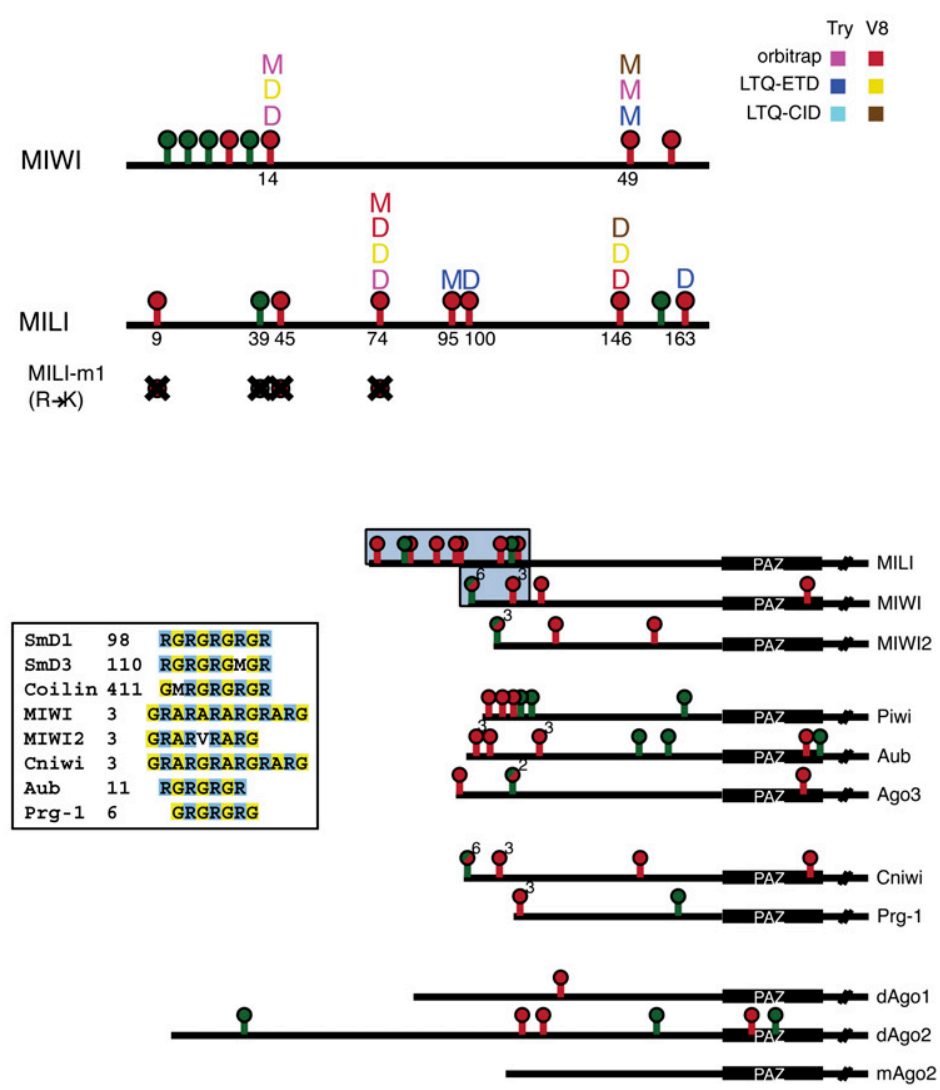

Figure 3. Mapping of arginine methylation sites in MIWI and MILI and conservation of arginine methylation sites among Piwi proteins. $(A)$ Potential arginine methylation sites (RA/RG motifs) are shown at N termini of MILI and MIWI (RA motifs, green; RG motifs, red). Monomethylation (M) and dimethylation (D) sites were identified by three different methods using digestion with trypsin or V8 protease and color coded accordingly. Below are shown positions of four arginine residues changed to lysine in the MILI-m1 construct. $(B)$ The $\mathrm{N}$ termini of murine (MIWI, MILI, and MIWI2), Drosophila (Piwi, Aub, and AGO3), cnidarian (Cniwi), and C. elegans (prg-1) Piwis and the Ago family members in Drosophila (dAgol and dAgo2) and mouse (mAgo2) are shown to scale. The positions of RA and RG motifs, marked as in $A$, are shown with stretches of multiple tandem motifs marked with the number of arginine residues. (Inset) Representative motifs in Piwis are aligned with known sites of symmetric arginine methylation in Sm D1, Sm D3, and p80 coilin. The boxed area of MILI and MIWI is shown in $A$. 
Vagin et al.

Piwi methylation is required for interaction with Tudor family proteins

The conservation of arginine methylation prompted a search for potential functions of such modifications. Tudor domains are known to recognize and bind to methylated arginines. For example, the Tudor domain within SMN binds methylarginine residues within Sm proteins as it chaperones snRNP assembly (MacKenzie and Gendron 2001). Analogously, symmetric dimethylarginine modification of p53 is essential for its interaction with 53BP1 (Kachirskaia et al. 2008). Notably, our proteomic analysis of 3xFlag-HA MILI, MIWI, and MIWI2 and of endogenous MIWI and MILI complexes revealed several proteins with single or multiple Tudor domains. We therefore investigated these as candidates for "readers" of the methylarginine marks within Piwi family members.

Piwi family immunoprecipitates contain six distinct Tudor domain proteins, TDRD1, TDRD2 (TDRKH), TDRD4 (RNF17), TDRD6, TDRD7, and TDRD9 either in adult or embryonic testes (Fig. 4). Each showed a discrete and specific pattern of interaction. Embryonic MILI complexes contained only TDRD1, while MIWI2 bound TDRD1, TDRD2, and to a much lesser extent, TDRD9 (Fig. 4D). MIWI associated with the greatest number of Tudor family proteins including TDRD1, TDRD2 and TDRD6 $(64,27$, and 136 peptides, representing $32.6 \%, 38.2 \%$, and 37.9\% coverage, respectively; Fig. 4C). To lesser degrees, MIWI complexes also contained TDRD4 (12 peptides, 8.7\% coverage), TDRD7, and TDRD9
A

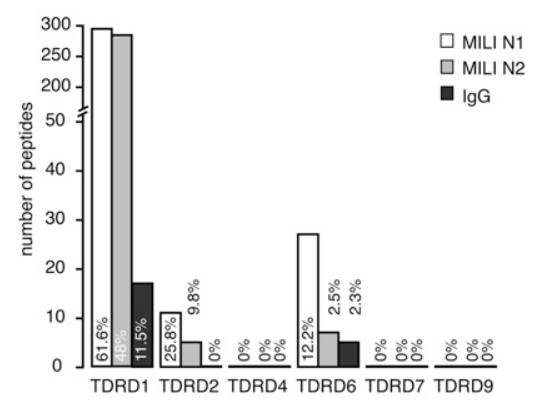

C

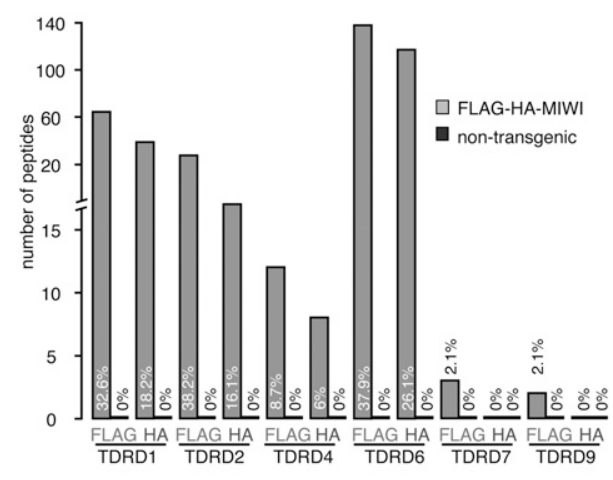

E

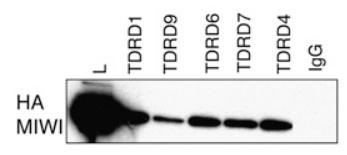

B

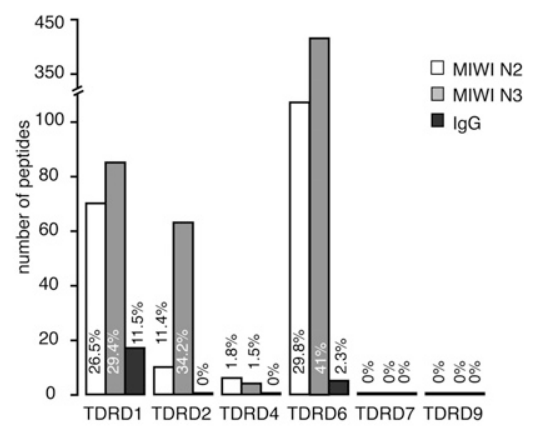

D
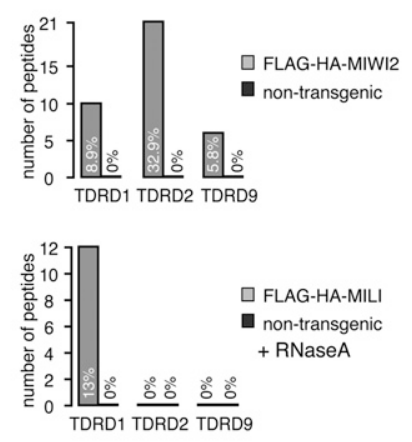

$\mathrm{F}$

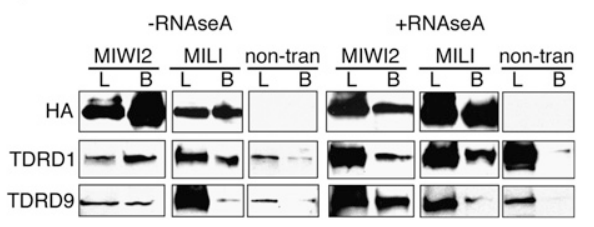

Figure 4. Piwi complexes with Tudor proteins. (A) Results of MudPIT analysis are shown for MILI complexes immunoprecipitated from adult testis extracts using MILI N1 or MILI N2 antibodies. The percent coverage is shown for each bar. $(B)$ Results of MudPIT analysis are shown for MIWI complexes immunoprecipitated from adult testis using MIWI N2 or MIWI N3 antibodies. (C) Results of MudPIT analysis are shown for immuoprecipitated 3xFlag-HA-MIWI complexes (adult testis). (D, top panel) Results of MudPIT analysis of immunoprecipitated 3xFlag-HA-MIWI2 complexes from E16.5-E18.5 testes are shown. (Bottom panel) Results of MudPIT analysis of immunoprecipitated 3xFlag-HA-MILI complexes from RNaseA-treated extracts are shown. (E) 3xFlag-HA-MIWI was immunoprecipitaed from adult testis extracts using TDRD1, TDRD9, TDRD4, TDRD6, TDRD7, or IgG antibodies and probed on Western with HA antibodies. (F) 3xFlag-HA-MIWI2 or 3xFlag-HA-MILI were immunoprecipitated from embryonic testes (E16.5-E18.5) using Flag beads and tested on Western with TDRD1, TDRD9 antibodies. (L:) Lysate; (B) bound fractions. 
(three and two peptides, $2.1 \%$ coverage). Interactions identified by mass spectrometry could be confirmed by Western blotting (for example, see Fig. 4E), and in no case did we note that interactions were sensitive to treatment of immunocomplexes with RNaseA (for example, see Fig. 4F).

Interactions between Piwi family members and Tudor domain proteins could be recapitulated in cultured, 293 cells. Upon coexpression, MILI and MIWI strongly interacted with TDRD1 (Fig. 5A). MIWI and MILI both interacted to differing degrees with TDRD2 and TDRD9. These results demonstrate that additional testis-specific factors are not required to mediate Piwi-Tudor interactions. Importantly, the interaction patterns observed in
A

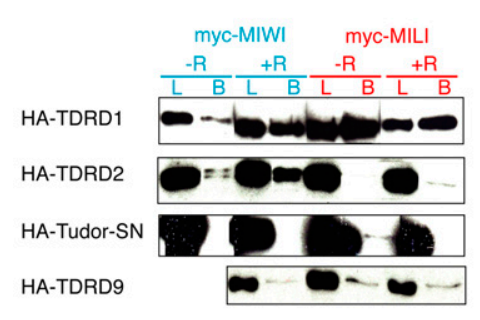

D

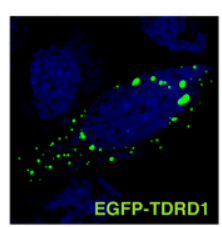

- TDRD1
B

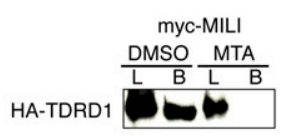

C
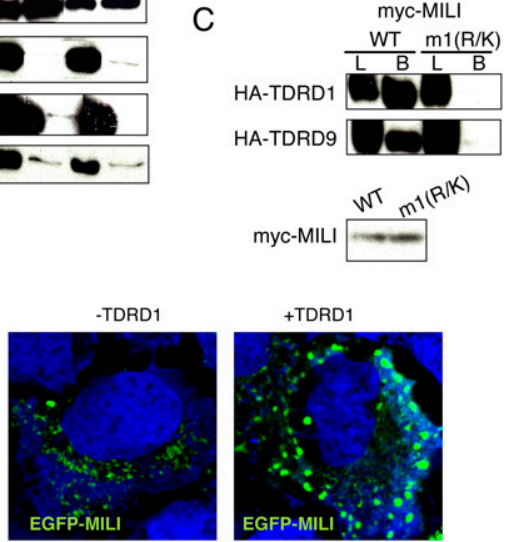

-TDRD1

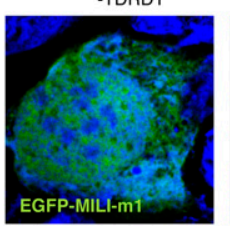

+TDRD1

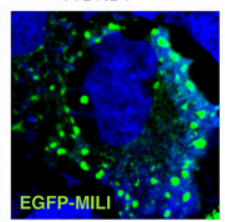

+TDRD1

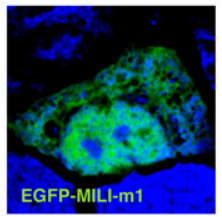

Figure 5. Tudors interacts with dimethylated arginines in Piwis. (A) Coimmunoprecipitation is shown for myc-MIWI or myc-MILI with Flag-HA-TDRD1, Flag-HA-TDRD2, Flag-HA-TDRD9, and Flag-HA-Tudor-SN from 293 cell extracts. Tagged MIWI or MILI proteins were immunoprecipitated from 293 cell with $(+\mathrm{R})$ or without $(-\mathrm{R})$ RNase A treatment using anti-myc beads and blotted using HA antibodies. (B) Coimmunoprecipitation is shown for myc-MILI with Flag-HA-TDRD1 from extracts of transfected 293 cells treated with an inhibitor of methylation (MTA). MILI was precipitated using anti-myc beads and probed with HA antibodies. (C, top panel) Coimmunoprecipitation is shown for Flag-HATDRD1 and TDRD9 with myc-MILI (WT) and myc-MILI-m1 (m1), where four arginine residues were substituted to lysine (see Fig. 3A). Tagged MILI proteins were immunoprecipitated from 293 cell extracts using anti-myc beads and probed with HA antibodies. (Bottom panel) The lysate, which was used for myc immunoprecipitation, was probed with myc antibodies. $(D)$ Patterns of ectopically expressed TDRD1, MILI, and MILI-m1 localization are shown for U2OS cells. Also shown are patterns of MILI and MILI-m1 in U2OS cells that express a nontagged TDRD1. germ cells are retained in this artificial system, suggesting that intrinsic features of these protein partners determine the specificity of complex formation.

Access to a cell culture model permitted us to test the determinants of Piwi-Tudor interactions. Ectopically expressed MIWI and MILI proteins become arginine methylated in 293 cells (Supplemental Fig. S5). Methylthioadenosine (MTA) is a relatively general inhibitor of methyltransferases. Treatment of transfected 293 cells with this compound prevented modification of MILI and MIWI. Coincidently, treatment with the inhibitor abolished interactions between MILI and TDRD1, implicating arginine methylation as a binding determinant for these proteins (Fig. 5B). The PRMT5/Wrd77 methylosome is associated with Piwi proteins in testis and is the only mammalian enzyme known to create the dimethylarginine marks observed on Piwi proteins. Thus, it is a prime candidate for mediating modifications of MILI and MIWI that are essential for Tudor binding.

To identify residues important for interaction between Piwi and Tudor family proteins, we focused on arginines in the MILI $\mathrm{N}$ terminus, since motifs in these positions are conserved. We prepared a quadruple mutant in which R9, R39, R45, and R74 were changed to lysine (MILI-m1 $[\mathrm{R} \rightarrow \mathrm{K}]$ ) (Fig. 3A). These were conservative substitutions that abolished a mapped methylation site (R74) on MILI in germ cells. The quadruple mutant showed no detectable binding to TDRD1 or TDRD9 in 293 cells (Fig. 5C). In parallel, we prepared a fusion protein in which the first 250 residues of MIWI were appended to an Ago2 protein lacking its first 174 amino acids. This MIWI fragment encompassed two of its three demonstrably methylated residues (R14 and $\mathrm{R} 49$ ). The fusion protein bound TDRD1, albeit somewhat more weakly than full-length MIWI (data not shown). No detectable binding was observed with full-length Ago2.

When transfected into 293 or U2OS cells, MILI is generally distributed in a finely granular pattern throughout the cytoplasm (Fig. 5D; data not shown). In contrast, TDRD1 collects in a number of large cytoplasmic foci. Coexpression with TDRD1 also causes MILI to accumulate in discrete foci. This depends on the integrity of mapped arginine methylation sites since the MILI-m1 mutant fails to adopt a TDRD1-dependent localization pattern.

Considered together, these results point to Tudor domain proteins as the readers of arginine methyl marks in Piwi proteins. Modification of arginine residues in the MILI N terminus is essential to mediate the interaction and fragments of MIWI-containing modification sites can confer binding on a heterologous fusion protein. The ability of TDRD1 to confer a focal localization pattern on MILI in an artificial context raised the possibility that arginine methylation and Tudor binding help to mediate assembly of piRNA pathway components into germline nuage.

\section{Colocalization of Tudor and Piwi proteins in nuage}

Just as with MIWI, MILI, and MIWI2, the integrity of a number of Tudor family proteins is essential for male 
fertility (Pan et al. 2005; Chuma et al. 2006; Vasileva et al. 2009; S Chuma, unpubl.). This provided a strong indication that their reading of arginine methyl marks and association with Piwi family members holds functional significance. Piwi family proteins occupy characteristic cytoplasmic domains, and their ability to localize to these structures has been linked to the efficient operation of the piRNA pathway (Lim and Kai 2007). We therefore probed the localization of Tudor family members and the interrelationships between these proteins and mammalian Piwis.

In haploid round spermatids, TDRD1, TDRD6, TDRD7, and TDRD9 all colocalize with MIWI in the chromatoid body (Fig. 6A). Notably, in meiotic spermatocytes, prior to the formation of the chromatoid body, these Tudor family members already occupy multiple cytoplasmic granules, which may represent chromatoid body precursors. In contrast, MIWI shows a more dispersed staining pattern at these stages. In Miwi-mutant animals, the chromatoid body localization of TDRD1, TDRD6, TDRD7, and TDRD9 is maintained (Supplemental Fig. S2). Considered together, these data support the notion that the recognition of methylated Piwis by Tudor family proteins might help to attract Piwi-RISC to chromatoid bodies or, alternatively, that such interactions might help to stabilize Piwi as a resident protein in these structures.

In the embryo, MIWI2 is expressed in prospermatogonia during the period of cell cycle arrest and establishment of de novo methylation patterns on repeat elements (Aravin et al. 2008; Kuramochi-Miyagawa et al. 2008). In these cell types, MIWI2 forms a ping-pong cycle with MILI to produce an efficient anti-transposon immune system (Aravin et al. 2008). This correlates with the localization of these proteins to distinct nuage compartments. In accord with previous studies, we did not detect expression of TDRD6 or TDRD7 in prospermatogonia (Hosokawa et al. 2007). However, TDRD1 and TDRD9 were expressed at this stage and localized to nuage (Fig. $6 \mathrm{~B}, \mathrm{C})$.

TDRD1 largely localizes in MILI-containing nuage, whereas TDRD9 is instead found in granules that contain MIWI2 (Fig. 6B; data not shown). TDRD9 was also found along with MIWI2 in gonocyte nuclei. These distinct patterns of localization paralleled patterns of specific biochemical interaction, where TDRD1 coimmunopreciptated with MILI and MIWI2 from embryonic testes, while TDRD9 associated with MIWI2.

In contrast to chromatoid bodies, where loss of MIWI does not impact the localization of several Tudor family proteins, mutations in Mili and Miwi2 did affect Tudor localization in embryonic testes. In Mili-deficient prospermatogonia, TDRD1 loses nuage localization and becomes dispersed in the cytoplasm. In contrast, MIWI2 expression is not required for correct TDRD1 localization (Fig. 6C). Loss of MILI also has dominant effects on both the localization of MIWI2 and its binding to piRNAs (Aravin et al. 2008). Coincidently, Mili-mutants also lose TDRD9 staining from both nuage and gonocyte nuclei (Fig. 6C). Despite their direct interaction and tight colo- calization, we saw a less dramatic, though detectable, impact of Miwi2 mutation on TDRD9 (Fig. 6C). This suggests that the impact of Mili loss on TDRD9 localization is not mediated solely through its documented effects on MIWI2 (Aravin et al. 2008). In fact, the impact of MILI on MIWI2 could be mediated in part by its effects on Tudor protein localization. In Tdrd1 mutants, MILI still occupied cytoplasmic nuage (Fig. 6D), and the nuclear localization of MIWI2 is retained. However, we noted a strong loss of MIWI2 from cytoplasmic nuage with a coincident appearance of diffuse cytoplasmic MIWI2 staining.

Overall, these results correlate patterns of localization with patterns of specific interactions between Piwi and Tudor family proteins. Moreover, they show interdependencies of localization to nuage compartments that are suggestive of functional relationships. Therefore, we sought to understand the functional relevance of TudorPiwi interactions for the piRNA pathway.

\section{TDRD1 is required for efficient operation of the ping-pong cycle}

In mammals, two separate piRNA pathways operate at distinct stages in germ cell development. In fetal gonocytes, MILI and MIWI2 form a ping-pong cycle that targets transposons for silencing, probably by both transcriptional and post-transcriptional mechanisms (Aravin et al. 2008; Kuramochi-Miyagawa et al. 2008). In meiotic germ cells, a complex population of piRNAs occupies MILI and MIWI, and this is depleted of transposon content in comparison with the genome as a whole (Aravin et al. 2006, 2008; Girard et al. 2006; Lau et al. 2006). To examine the function of Tudor proteins in germ cells, we examined the impact of Tudor mutants on the piRNA pathway.

TDRD6 is expressed only postnatally (data not shown). We therefore cloned piRNAs from testis of 18-d-old (prior to the onset of any observable phenotype), 25-d-old (as spermatogenic defects are becoming apparent), and 6-wkold animals, either from Tdrd6 mutants or their heterozygous littermates (S Chuma, unpubl.). At all three stages examined, we noted no or only very minor impacts on either piRNA or miRNA profiles. Recent studies have suggested a moderate effect of TDRD6 loss on miRNA expression (Vasileva et al. 2009). We did find that P18 and P25 animals had lower levels of miRNAs as compared with piRNAs (3.6-fold reduction overall). However, the fraction and content of miRNA populations in P25 Tdrd6 mutants was similar to that observed in 6-wk-old heterozygotes (data not shown), suggesting that the impact of Tdrd6 lesions might be on developmental timing rather than on the miRNA pathway, per se. The overall lack of an impact on piRNAs in the meiotic cells of this mutant could reflect redundant functions of multiple family members that are coexpressed, colocalize, and all bind to Piwi family members at this developmental stage.

In contrast, loss of TDRD1 did impact piRNA populations. In this case, we focused on prospermatogonia and the piRNAs that control mobile elements. Total small 
A
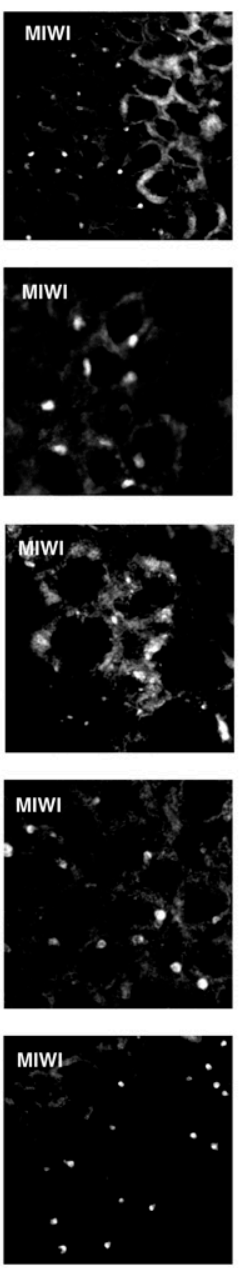

B

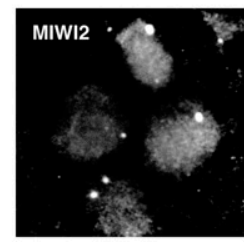

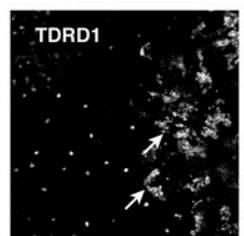
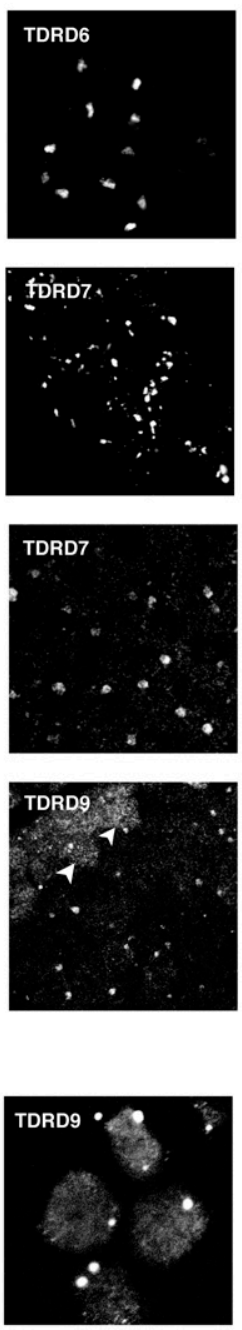
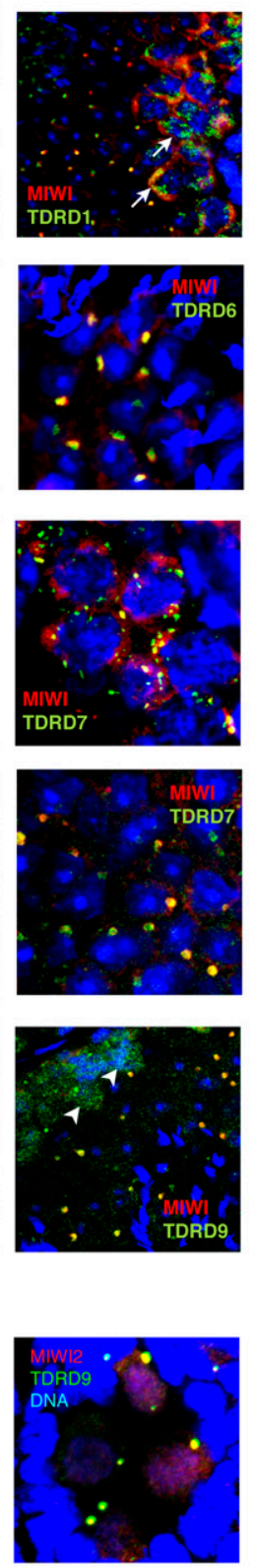

C
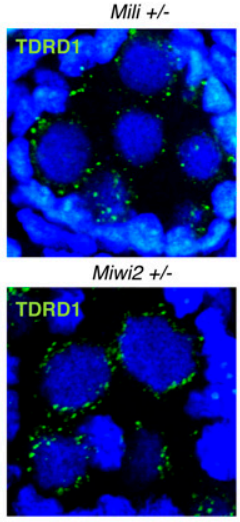

Mili +/-

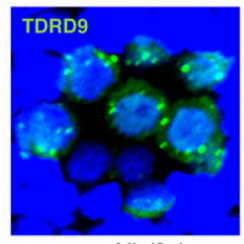

Miwi2+/-

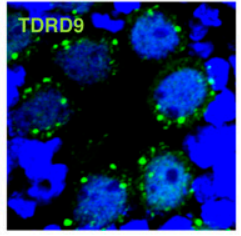

D
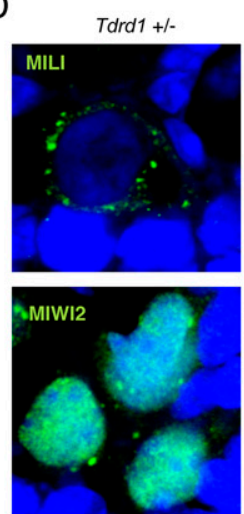

Mili -\%

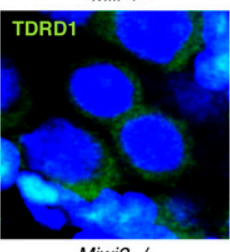

Miwi2 -\%

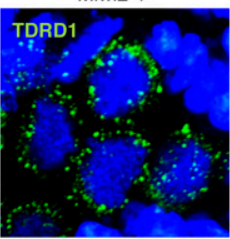

Mili -/-

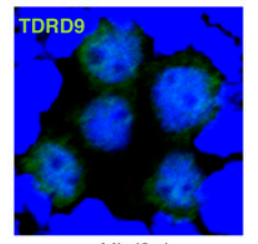

Miwi2 -/-

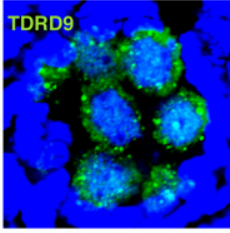

Tdrd1 \%
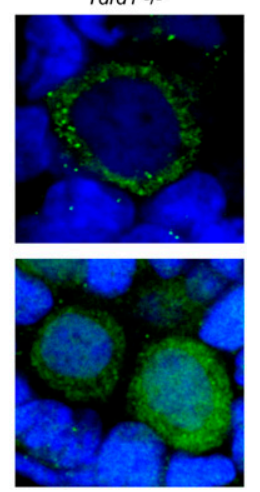

Figure 6. Colocalization and interdependence of Piwi and Tudor proteins in cytoplasmic nuage. (A) Localization of TDRD1, TDRD6, TDRD7, TDRD9 and MIWI are shown in testes of adult (2-mo-old) mice expressing the myc-MIWI transgene. Four Tudor proteins and MIWI colocalize in a single nuage granule, the chromatoid body, in round spermatids. TDRD1 and TDRD7 localize to numerous small nuage granules at earlier stages, primary spermatocytes (arrow), while MIWI is more dispersed in the cytoplasm at this stage. Note the nuclear localization of TDRD9 in spermatogonia and primary spermatocytes (arrowhead). (B) Localization of MIWI2 and TDRD9 in embryonic (E17.5) testes shows a complete colocalization of these proteins in a few cytoplasmic granules in prospermatogonia. $(C)$ The effect of MILI and MIWI2 deficiency on TDRD1 and TDRD9 localization is shown for E17.5 prospermatogonia. $(D)$ The effect of TDRD1 deficiency on MILI and MIWI2 localization is shown for E17.5 prospermatogonia.

RNA populations (18-33 nucleotide [nt]) were isolated from embryonic (E18.5) testis from Tdrd1 mutants and their heterozygous littermates. Following Illumina sequencing, small RNAs were split into miRNAs (21-23 nt) and piRNAs (25-33 nt). While we saw no impact on miRNAs in the mutants, piRNAs were substantially reduced. Following normalization to miRNAs, we noted 5.2-fold fewer piRNAs in Tdrd1 mutants, as compared with controls (Fig. 7A). Repeat-derived piRNAs were still present in mutant animals, although the levels of species that target prominent, potentially active transposon classes were strongly impacted.

MIWI2 and MILI associate with piRNA populations that can be discriminated in several ways. MILI binds 
Vagin et al.

A

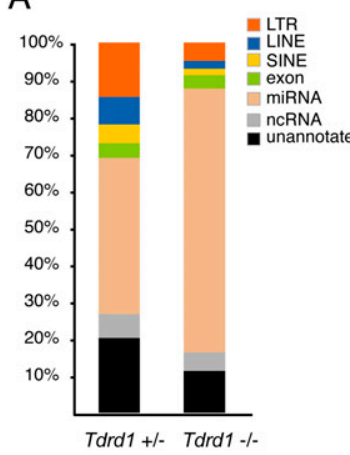

C

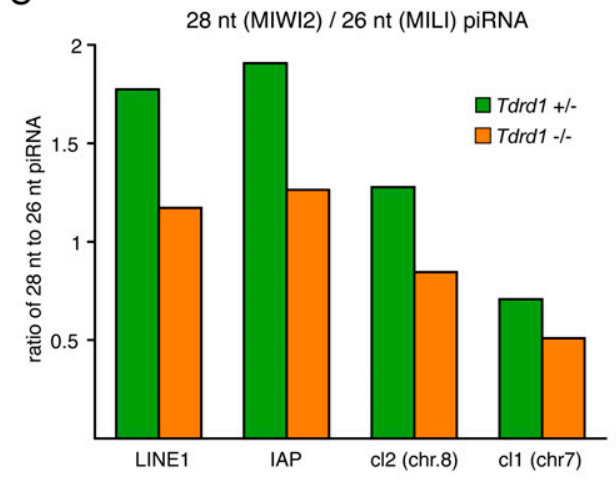

$\mathrm{D}$

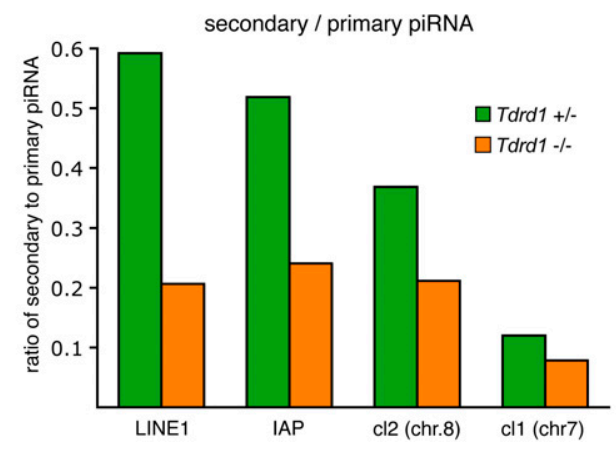

B
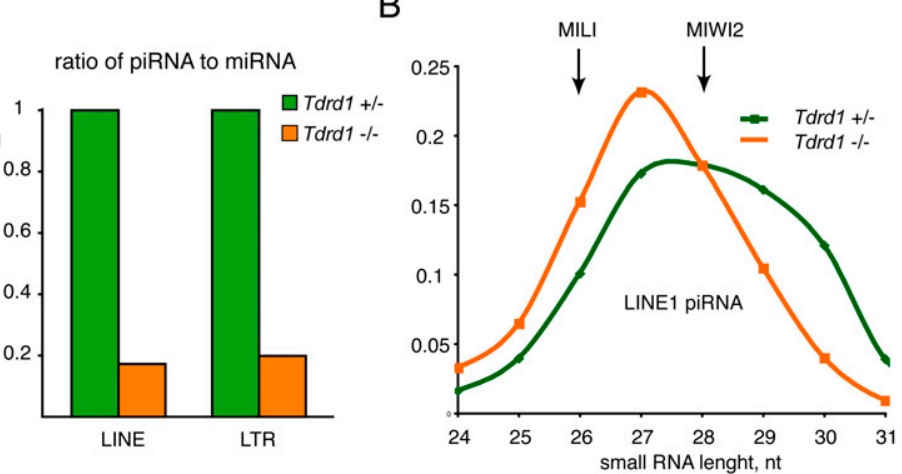

E
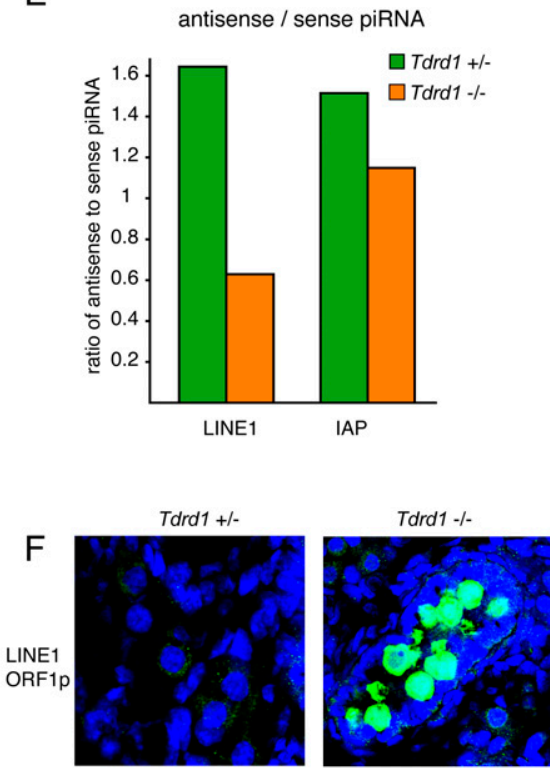

IAP

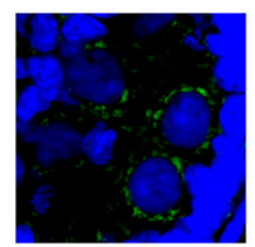

Figure 7. The effect of TDRD1 deficiency on piRNA expression in prospermatogonia. (A) Annotation is shown for small RNAs cloned from embryonic (E18.5) testes of Tdrd1 heterozygous and homozygous knockout $(\mathrm{KO})$ animals. $(B)$ Total cellular piRNA populations are composed of two complexes, MILI, with an average piRNA length $26 \mathrm{nt}$ and MIWI2 with an average piRNA length $28 \mathrm{nt}$. The size profile reflects the ratio of both complexes in the cell. (C) The ratio of 28-nt (MIWI2) to 26-nt (MILI) piRNAs that correspond to LINE1 and IAP retrotransposons as well as single-stranded (cl1, chr.7) and double-stranded (cl2, chr.8) piRNA clusters decreases in the Tdrd1 mutant. $(D)$ The fraction of secondary piRNAs matching retrotransposons and piRNA clusters decreases in Tdrd1 mutants. $(E)$ The fraction of antisense piRNAs that correspond to L1 and IAP retrotransposons is reduced in Tdrd1 knockouts. $(F)$ Localization of LINE1and IAP-encoded proteins in testes of E17.5 heterozygous and homozygous Tdrd1 animals. Pictures of knockout and control animals were taken with identical exposure levels.

smaller RNAs ( 26 nt), while those occupying MIWI2 tend to be longer $(\sim 28 \mathrm{nt})$. Moreover, MIWI2 is enriched for secondary piRNAs that tend to be antisense to transposons (Aravin et al. 2008). By examining piRNA size profiles (Fig. 7B), we noted selective impacts of TDRD1 loss on MIWI2-bound piRNA populations. This was true whether we focused on piRNAs targeting specific transposon classes or on uniquely mapping piRNAs derived from specific generative loci (Fig. 7C). Consistent with our prevailing model of the operation of the piRNA pathway in fetal gonocytes, this translated into a selective loss of antisense piRNAs with the potential to regulate and suppress active transposons (Fig. 7E) and with a reduction in secondary piRNA species (Fig. 7D).

In agreement with its impacts on piRNAs, loss of TDRD1 affected transposon silencing. Mutant animals 
showed a strong activation of LINE1 ORF1p expression (Fig. 7F). However, silencing of IAP remained relatively robust, despite equivalent impacts on piRNAs targeting both transposon classes. This might simply indicate that IAP silencing can tolerate greater impacts on the operation of the ping-pong cycle than can L1 while still retaining repression.

Considered together, these data suggest a functional role for the Tudor family protein, TDRD1, in the piRNA pathway. The specific nature of the impact of TDRD1 loss on piRNA populations correlates well with its selective impact on MIWI2 localization to cytoplasmic nuage and provide further support for the importance of this compartmentalization to optimal function of the transposon silencing machinery.

\section{Discussion}

Our comprehensive proteomic analysis of complexes containing mouse Piwi proteins, MIWI, MILI, and MIWI2, has revealed a new motif in mammalian transposon control formed by discrete axes of interaction between Piwi and Tudor domain-containing proteins. Previous studies have implicated Tudor domain proteins in RISC- or small RNA-related pathways. For example, a Tudor domaincontaining nuclease Tudor-SN was identified as a component of Ago-RISC in Drosophila cells, with functional relationships to siRNA-mediated silencing in C. elegans (Caudy et al. 2003). Spindle-E, the Drosophila ortholog of TDRD9, interacts with Piwi family proteins and plays critical roles in the piRNA pathway in germ cells (Aravin et al. 2001, 2004; Vagin et al. 2006; Malone et al. 2009). During the preparation of this report, two groups noted interactions between TDRD1 and MILI and TDRD6 and MIWI, though neither the determinants nor functional consequences of complex formation were investigated (Vasileva et al. 2009; J Wang et al. 2009). Copurification of a Tudor protein and Aubergine has also been reported in Drosophila (Thomson et al. 2008). Here, we report pervasive interactions between specific Tudor domain proteins and Piwi family-binding partners in mice. These biochemical interactions are specific and are reflected at the level of developmental coexpression and patterns of colocalization to distinct nuage structures. Since patterns of specific interaction can be reproduced in ectopic settings in which no piRNA pathway operates and in which no nuage structures form, the specificity of interaction observed in germ cells are not simply a consequence of propinquity but instead must reflect intrinsic differences in affinity among different Tudor-Piwi pairs.

Tudor proteins recognize symmetrically dimethylated arginine and lysine residues (Cote and Richard 2005). Such modifications mediate interaction between SMN and Sm proteins, p53 and 53BP1, and between JMJD2A, 53BP1, and histone H3 (Huyen et al. 2004; Huang et al. 2006; Kachirskaia et al. 2008; Zgheib et al. 2009). Our proteomic analyses indicated that all three mammalian Piwi family members complex with the PRMT5/WDR77 methylosome. During the preparation of this manuscript, arginine methylation of Aub and Ago3 by Capsuleen was also noted in flies (Kirino et al. 2009). Piwi family proteins, but not members of the Ago clade, contained conserved runs of RG/RA in their $\mathrm{N}$ termini that resemble known sites of recognition by the methylosome complex. Based on a series of analyses, we were able to demonstrate the presence of both arginine monomethylation and symmetric dimethylation in MIWI and MILI. Detailed mapping of methylation sites indicated modification of conserved $\mathrm{N}$-terminal residues. Through the use of both relatively general methylation inhibitors and through directed mutagenesis, we were able to demonstrate that formation of specific Tudor domain-Piwi family interactions depended wholly on both methylation and the presence of specific target arginine residues. Thus, our evidence supports the notion that Tudor domain proteins read out a methylarginine code-perhaps similar to the histone code- that is written on Piwi family members by PRMT5 and perhaps also other methyltransferases.

The functional relevance of this code is supported by genetic experiments in which disruption of the piRNA pathway impacts Tudor localization and reciprocally loss of Tudor proteins impact both the localization and function of Piwi proteins. Loss of TDRD1 has strong impacts on both the piRNA pathway and the localization of its components in gonocytes. Moreover, TDRD1 can bring MILI to discrete cytoplasmic foci in somatic cell lines. In contrast, loss of TDRD6 had minimal impacts on small RNA populations in meiotic germ cells, though this mutation has been reported to cause loss of MIWI from the chromatoid body (Vasileva et al. 2009). Reciprocally, loss of MIWI failed to impact localization of multiple Tudor proteins in the chromatoid body. The genetic interdependencies (or lack thereof) that we observe might reflect partial redundancy of Piwi proteins and the fact that Piwis are probably not the only binding partners of these Tudor family members in germ cells. In this regard, it was recently demonstrated that TDRD6 interacts with MVH (Vasileva et al. 2009), likely reflecting methylation of conserved arginine residues in the MVH $\mathrm{N}$ terminus (data not shown). Indeed, MVH localizes in nuage granules in prospermatogonia as well as in the chromatoid body in round spermatids and is required for TDRD1, TDRD6, and TDRD7 localization (Hosokawa et al. 2007).

Inquiries into the functions of Tudor domain proteins such as SMN suggest that these can act as chaperones for the assembly of macromolecular proteins complexes (Brahms et al. 2001). The large number of Tudor domain proteins involved, the complexity of their interaction patterns, and the interrelationships between the function and localization of Tudor and Piwi family members suggests a general role for arginine methylation and Tudor proteins in maintaining the integrity of small RNA pathways critical to germ cell preservation. Even ectopic expression of a single Tudor domain could disrupt the localization of chromatoid body components in round spermatids, suggesting that Tudor proteins could act as more general determinants of assembly of germline nuage (Hosokawa et al. 2007). In some cases, Tudor-proteins appear critical for the localization of methylated compartment components to nuage and there appear to be 
reciprocal requirements for methylated proteins to bring Tudors to their native cellular locales. An example in Drosophila is Vasa, which acts upstream of Tudor in the pathway that assembles the pole plasm. Our results suggest that MILI is important for localization of TDRD9 to nuage and, conversely, that TDRD1 is critical for localization of MIWI2 to these same structures.

An emerging theme that is highlighted by the present work is that germ cells depend critically on compartmentalization of small RNA pathways. While our studies reflect this in the construction of the piRNA pathway, the observation of Argonaute-containing granules in other systems-for example, P-granules in C. elegans (Batista et al. 2008) —and the recently demonstrated roles of small RNAs as mediators of epigenetic inheritance in Drosophila (Brennecke et al. 2008) underscore the need for a tightly orchestrated and highly regulated pathway of RNP granule assembly. Our data point to important roles for arginine methylation and Tudor family proteins in these processes.

\section{Materials and methods}

\section{Animals and transgenic constructs}

The Miwi, Miwi2, and Mili knockout strains were described in Deng and Lin (2002), Carmell et al. (2007), and KuramochiMiyagawa et al. (2004), respectively. 3xmyc-MIWI2, 3xmycMIWI, and 3xFlag-HA-MILI, 3xFlag-HA-MIWI and 3xFlag-HAMIWI2 transgenic animals were described previously or produced as described previously (Aravin et al. 2008).

\section{Immunofluorescence detection of protein localization}

Immunofluorescence detection of protein in paraffin-embedded testicular sections was performed for 3xmyc-MIWI2, 3xmycMIWI, Mili, and Miwi2 knockout animals and respective controls as described previously (Aravin et al. 2008). For Tdrd1 knockout and control animals, frozen sections were used (see the Supplemental Material).

\section{Small RNA libraries}

Small RNA isolation, library construction, and annotation were performed as described previously (Aravin et al. 2007b)

\section{Antibodies}

Antibodies were LINE1 Orf1p (1:500; S.L. Martin), IAP (1:1000; B.R. Cullen), MILI (1:100) (Aravin et al. 2007b), MIWI2 (1:750) (Aravin et al. 2008), Mael (1:1000) (Soper et al. 2008), TDRD1, TDRD6, TDRD7 (1:1000) (Hosokawa et al. 2007), TDRD9 (1:1000; S Chuma, unpubl.), MVH (1:1000; Abcam ab13840), GW182 (1:50; M. Fritzler), myc (1:300; Upstate Biotechnologies clone 4A6), PRMT5 (1:100; Abcam ab31751).

\section{MudPIT and protein modification analysis}

For MudPIT analysis, endogenous MIWI and MILI complexes were recovered from testis of 3- to 6-mo-old C57BL/6 animals. For immunoprecipitation of Flag-HA-tagged MIWI, MILI, and MIWI2 complexes, transgenic animals were used; nontransgenic CD-1 animals were used as controls. Immunoprecipitations of Flag-HA-MIWI2 and MILI complexes were performed from 16.5- to18.5-d-old embryos obtained from crosses of CD-1 nontransgenic females with Flag-HA-MIWI2 or Flag-HA-MILI transgenic males. See the Supplemental Material for details of the immunoprecipitation and sample preparation protocols.

Protein modifications were identified by Nano-LC/Orbitrap CID/ETD as described in the Supplemental Material.

Coimmunoprecipitation of Flag-HA-MIWI, MILI, and MIWI2 with Tudors, PRMT5, and WDR77 from testis lysates

Testes were dissected from Flag-HA-MIWI, MILI, or MIWI2 transgenic animals. Procedures for Flag precipitation were essentially as described for MudPIT analysis, except that beads were washed once with NT2 buffer (containing $150 \mathrm{mM} \mathrm{NaCl}$ ), once with NT2 buffer (containing $300 \mathrm{mM} \mathrm{NaCl}$ ), once with $\mathrm{NT} 2$ buffer (containing $450 \mathrm{mM} \mathrm{NaCl}$ ) for $10 \mathrm{~min}$ each at $4^{\circ} \mathrm{C}$. To elute Flag-HA-PIWI complexes the beads were mixed with equal volume of protein Laemmli buffer and incubated for $10 \mathrm{~min}$ at $95^{\circ} \mathrm{C}$. Samples were spun and the supernatant was collected.

Precipitation of Tudor, PRMT5, and WDR77 complexes was done essentially as described for endogenous MIWI and MILI MudPIT analysis. At the last step the complexes were eluted with Laemmli buffer. The following antibodies were used for immunoprecipitation at a 1:100 dilution: TDRD1, TDRD6, TDRD7 (Hosokawa et al. 2007), TDRD4 (antiserum 1774, (Pan et al. 2005), TDRD9 (S Chuma, unpubl.), SYM10 (07-412, Millipore), WDR77 (A301-561A, Bethyl), PRMT5 (07-405, Millipore) and rabbit IgG (12-370, Millipore).

For Western blotting we used antibodies to TDRD1, TDRD6, TDRD7 (Hosokawa et al. 2007), TDRD4 (antiserum 1774) (Pan et al. 2005), TDRD9 (S Chuma, unpubl.), SYM10 (07-412, Millipore), WDR77 (A301-561A, Bethyl), PRMT5 (07-405, Millipore) at a 1:800 dilution. To detect Flag-HA-PIWIs we used 1:2000 HAHRP antibodies (1:500; Roche, 12013819001); for the rest of the proteins analyzed secondary antibody were 1:3000 anti-mouseHRP or anti-rabbit-HRP (\#7076 and \#7074, Cell Signaling).

MIWI and Tudor coimmunoprecipitation and drug treatment in 293 cells

293 cells were cotransfected with myc-PIWIs and Flag-HATudors or Flag-HA-PRMT5, Flag-HA-WDR77 plasmids. Cell lysates and immunoprecipitation were performed as described for Flag-HA-MIWI precipitation from testes. Anti-myc immunoprecipitation was performed using $20 \mu \mathrm{L}$ of 9E10 monoclonal antibody matrix (AFC-150P, Covance) according to the manufacturer's protocol.

5'-Deoxy-5'-(methylthio)-adenosine (MTA, $750 \mu \mathrm{M})$ (D5011100MG, Sigma) treatment was done $1 \mathrm{~d}$ prior to cotransfection with Flag-HA-TDRD1 and myc-MILI.

\section{Acknowledgments}

We thank S.L. Martin, J. Wang, B.R. Cullen, A. Bortvin, and M. Fritzler for sharing antibodies, and N. Nakatsuji, T. Tanaka, and M. Hosokawa for tissues and Tudor mutant animals. We thank Sang Yong Kim (CSHL) for generating transgenic animals. We thank Maria Mosquera, Lisa Bianco, Jodi Coblentz, and Gula Nourjanova (CSHL) for animal assistance and histology, and Michelle Rooks, Dick Mccombie, Danea Rebbolini, and Laura Cardone for help with Illumina sequencing. We thank Katalin Fejes Toth, Nikolay Rozhkov, and Antoine Molaro for help with experiments and illustrations, and Assaf Gordon for help with data analysis. S.C. is supported by Grants-in-Aid from MEXT, Japan; J.W. was supported by funds from the Jonsson Cancer 
Center at UCLA; and J.Q. is supported by the University at Buffalo Center of Protein Therapeutics grant. G.J.H. is an investigator of the Howard Hughes Medical Institute. This work was supported by a kind gift from Kathryn W. Davis and grants from the National Institutes of Health to G.J.H., and an NIH Pathway to Independence Award K99HD057233 to A.A.A.

\section{References}

Aravin AA, Naumova NM, Tulin AV, Vagin VV, Rozovsky YM, Gvozdev VA. 2001. Double-stranded RNA-mediated silencing of genomic tandem repeats and transposable elements in the D. melanogaster germline. Curr Biol 11: 10171027.

Aravin AA, Klenov MS, Vagin VV, Bantignies F, Cavalli G, Gvozdev VA. 2004. Dissection of a natural RNA silencing process in the Drosophila melanogaster germ line. Mol Cell Biol 24: 6742-6750.

Aravin A, Gaidatzis D, Pfeffer S, Lagos-Quintana M, Landgraf $P$, Iovino N, Morris P, Brownstein MJ, Kuramochi-Miyagawa S, Nakano T, et al. 2006. A novel class of small RNAs bind to MILI protein in mouse testes. Nature 442: 203-207.

Aravin AA, Hannon GJ, Brennecke J. 2007a. The Piwi-piRNA pathway provides an adaptive defense in the transposon arms race. Science 318: 761-764.

Aravin AA, Sachidanandam R, Girard A, Fejes-Toth K, Hannon GJ. 2007b. Developmentally regulated piRNA clusters implicate MILI in transposon control. Science 316: 744-747.

Aravin AA, Sachidanandam R, Bourc'his D, Schaefer C, Pezic D, Toth KF, Bestor T, Hannon GJ. 2008. A piRNA pathway primed by individual transposons is linked to de novo DNA methylation in mice. Mol Cell 31: 785-799.

Batista PJ, Ruby JG, Claycomb JM, Chiang R, Fahlgren N, Kasschau KD, Chaves DA, Gu W, Vasale JJ, Duan S, et al. 2008. PRG-1 and 21U-RNAs interact to form the piRNA complex required for fertility in C. elegans. Mol Cell 31: 6778.

Brahms H, Meheus L, de Brabandere V, Fischer U, Luhrmann R. 2001. Symmetrical dimethylation of arginine residues in spliceosomal $\mathrm{Sm}$ protein $\mathrm{B} / \mathrm{B}^{\prime}$ and the $\mathrm{Sm}$-like protein LSm4, and their interaction with the SMN protein. RNA 7: 1531-1542.

Brennecke J, Aravin AA, Stark A, Dus M, Kellis M, Sachidanandam R, Hannon GJ. 2007. Discrete small RNA-generating loci as master regulators of transposon activity in Drosophila. Cell 128: 1089-1103.

Brennecke J, Malone CD, Aravin AA, Sachidanandam R, Stark A, Hannon GJ. 2008. An epigenetic role for maternally inherited piRNAs in transposon silencing. Science 322: 1387-1392.

Brower-Toland B, Findley SD, Jiang L, Liu L, Yin H, Dus M, Zhou P, Elgin SC, Lin H. 2007. Drosophila PIWI associates with chromatin and interacts directly with HPla. Genes \& Dev 21: 2300-2311.

Carmell MA, Girard A, van de Kant HJ, Bourc'his D, Bestor TH, de Rooij DG, Hannon GJ. 2007. MIWI2 is essential for spermatogenesis and repression of transposons in the mouse male germline. Dev Cell 12: 503-514.

Caudy AA, Ketting RF, Hammond SM, Denli AM, Bathoorn AM, Tops BB, Silva JM, Myers MM, Hannon GJ, Plasterk RH. 2003. A micrococcal nuclease homologue in RNAi effector complexes. Nature 425: 411-414.

Chendrimada TP, Gregory RI, Kumaraswamy E, Norman J, Cooch N, Nishikura K, Shiekhattar R. 2005. TRBP recruits the Dicer complex to Ago2 for microRNA processing and gene silencing. Nature 436: 740-744.
Chuma S, Hosokawa M, Kitamura K, Kasai S, Fujioka M, Hiyoshi M, Takamune K, Noce T, Nakatsuji N. 2006. Tdrd1/Mtr-1, a tudor-related gene, is essential for male germ-cell differentiation and nuage/germinal granule formation in mice. Proc Natl Acad Sci 103: 15894-15899.

Cote J, Richard S. 2005. Tudor domains bind symmetrical dimethylated arginines. J Biol Chem 280: 28476-28483.

Das PP, Bagijn MP, Goldstein LD, Woolford JR, Lehrbach NJ, Sapetschnig A, Buhecha HR, Gilchrist MJ, Howe KL, Stark R, et al. 2008. Piwi and piRNAs act upstream of an endogenous siRNA pathway to suppress Tc3 transposon mobility in the Caenorhabditis elegans germline. Mol Cell 31: 79-90.

Deng W, Lin H. 2002. miwi, a murine homolog of piwi, encodes a cytoplasmic protein essential for spermatogenesis. Dev Cell 2: 819-830.

Duan X, Young R, Straubinger R, Page B, Cao J, Wang H, Yu H, Canty J, Qu J. 2009. A straightforward and highly efficient precipitation/on-pellet digestion procedure coupled to long gradient nano-LC separation and Oribtrap mass spectrometry for the label-free expression profiling of swine heart mitochondria proteome. J Proteome Res 8: 2838-2850.

Friesen WJ, Wyce A, Paushkin S, Abel L, Rappsilber J, Mann M, Dreyfuss G. 2002. A novel WD repeat protein component of the methylosome binds Sm proteins. J Biol Chem 277: 82438247.

Girard A, Sachidanandam R, Hannon GJ, Carmell MA. 2006. A germline-specific class of small RNAs binds mammalian Piwi proteins. Nature 442: 199-202.

Gregory RI, Chendrimada TP, Cooch N, Shiekhattar R. 2005. Human RISC couples microRNA biogenesis and posttranscriptional gene silencing. Cell 123: 631-640.

Gunawardane LS, Saito K, Nishida KM, Miyoshi K, Kawamura Y, Nagami T, Siomi H, Siomi MC. 2007. A slicer-mediated mechanism for repeat-associated siRNA $5^{\prime}$ end formation in Drosophila. Science 315: 1587-1590.

Hosokawa M, Shoji M, Kitamura K, Tanaka T, Noce T, Chuma S, Nakatsuji N. 2007. Tudor-related proteins TDRD1/MTR-1, TDRD6 and TDRD7/TRAP: Domain composition, intracellular localization, and function in male germ cells in mice. Dev Biol 301: 38-52.

Houwing S, Kamminga LM, Berezikov E, Cronembold D, Girard A, van den Elst H, Filippov DV, Blaser H, Raz E, Moens CB, et al. 2007. A role for Piwi and piRNAs in germ cell maintenance and transposon silencing in Zebrafish. Cell 129: 6982.

Houwing S, Berezikov E, Ketting RF. 2008. Zili is required for germ cell differentiation and meiosis in zebrafish. EMBO J 27: 2702-2711.

Huang Y, Fang J, Bedford MT, Zhang Y, Xu RM. 2006. Recognition of histone $\mathrm{H} 3$ lysine-4 methylation by the double tudor domain of JMJD2A. Science 312: 748-751.

Hutvagner G, Simard MJ. 2008. Argonaute proteins: Key players in RNA silencing. Nat Rev Mol Cell Biol 9: 22-32.

Huyen Y, Zgheib O, Ditullio RA Jr, Gorgoulis VG, Zacharatos P, Petty TJ, Sheston EA, Mellert HS, Stavridi ES, Halazonetis TD. 2004. Methylated lysine 79 of histone H3 targets 53BP1 to DNA double-strand breaks. Nature 432: 406-411.

Jansson M, Durant ST, Cho EC, Sheahan S, Edelmann M, Kessler B, La Thangue NB. 2008. Arginine methylation regulates the p53 response. Nat Cell Biol 10: 1431-1439.

Kachirskaia I, Shi X, Yamaguchi H, Tanoue K, Wen H, Wang EW, Appella E, Gozani O. 2008. Role for 53BP1 Tudor domain recognition of p53 dimethylated at lysine 382 in DNA damage signaling. I Biol Chem 283: 34660-34666.

Kirino Y, Kim N, de Planell-Saguer M, Khandros E, Chiorean S, Klein PS, Rigoutsos I, Jongens TA, Mourelatos Z. 2009. 
Vagin et al.

Arginine methylation of Piwi proteins catalysed by dPRMT5 is required for Ago3 and Aub stability. Nat Cell Biol 11: 652658.

Kotaja N, Lin H, Parvinen M, Sassone-Corsi P. 2006. Interplay of PIWI/Argonaute protein MIWI and kinesin KIF17b in chromatoid bodies of male germ cells. I Cell Sci 119: 28192825.

Kuramochi-Miyagawa S, Kimura T, Ijiri TW, Isobe T, Asada N, Fujita Y, Ikawa M, Iwai N, Okabe M, Deng W, et al. 2004. Mili, a mammalian member of piwi family gene, is essential for spermatogenesis. Development 131: 839-849.

Kuramochi-Miyagawa S, Watanabe T, Gotoh K, Totoki Y, Toyoda A, Ikawa M, Asada N, Kojima K, Yamaguchi Y, Ijiri TW, et al. 2008. DNA methylation of retrotransposon genes is regulated by Piwi family members MILI and MIWI2 in murine fetal testes. Genes \& Dev 22: 908-917.

Lau NC, Seto AG, Kim J, Kuramochi-Miyagawa S, Nakano T, Bartel DP, Kingston RE. 2006. Characterization of the piRNA complex from rat testes. Science 313: 363-367.

Lim AK, Kai T. 2007. Unique germ-line organelle, nuage, functions to repress selfish genetic elements in Drosophila melanogaster. Proc Natl Acad Sci 104: 6714-6719.

Liu J, Rivas FV, Wohlschlegel J, Yates JR 3rd, Parker R, Hannon GJ. 2005. A role for the P-body component GW182 in microRNA function. Nat Cell Biol 7: 1261-1266.

MacKenzie AE, Gendron NH. 2001. Tudor reign. Nat Struct Biol 8: $13-15$

Malone CD, Brennecke J, Dus M, Stark A, McCombie WR, Sachidanandam R, Hannon GJ. 2009. Specialized piRNA pathways act in germline and somatic tissues of the Drosophila ovary. Cell 137: 522-535.

Meister G, Landthaler M, Peters L, Chen PY, Urlaub H, Luhrmann R, Tuschl T. 2005. Identification of novel argonaute-associated proteins. Curr Biol 15: 2149-2155.

Ohtsuka M, Kimura M, Tanaka M, Inoko H. 2009. Recombinant DNA technologies for construction of precisely designed transgene constructs. Curr Pharm Biotechnol 10: 244-251.

Pal S, Vishwanath SN, Erdjument-Bromage H, Tempst P, Sif S. 2004. Human SWI/SNF-associated PRMT5 methylates histone $\mathrm{H} 3$ arginine 8 and negatively regulates expression of ST7 and NM23 tumor suppressor genes. Mol Cell Biol 24: 9630-9645.

Pan J, Goodheart M, Chuma S, Nakatsuji N, Page DC, Wang PJ. 2005. RNF17, a component of the mammalian germ cell nuage, is essential for spermiogenesis. Development 132: 4029-4039.

Saito K, Nishida KM, Mori T, Kawamura Y, Miyoshi K, Nagami T, Siomi H, Siomi MC. 2006. Specific association of Piwi with rasiRNAs derived from retrotransposon and heterochromatic regions in the Drosophila genome. Genes \& Dev 20: 2214-2222.

Soper SF, van der Heijden GW, Hardiman TC, Goodheart M, Martin SL, de Boer P, Bortvin A. 2008. Mouse maelstrom, a component of nuage, is essential for spermatogenesis and transposon repression in meiosis. Dev Cell 15: 285-297.

Thomson T, Liu N, Arkov A, Lehmann R, Lasko P. 2008. Isolation of new polar granule components in Drosophila reveals P body and ER associated proteins. Mech Dev 125: 865-873.

Vagin VV, Sigova A, Li C, Seitz H, Gvozdev V, Zamore PD. 2006. A distinct small RNA pathway silences selfish genetic elements in the germline. Science 313: 320-324.

Vasileva A, Tiedau D, Firooznia A, Muller-Reichert T, Jessberger R. 2009. TDR: $\mathrm{d} 6$ is required for spermiogenesis, chromatoid body architecture, and regulation of miRNA expression. Curr Biol 19: 630-639.
Wang H, Straubinger RM, Aletta JM, Cao J, Duan X, Yu H, Qu J. 2009. Accurate localization and relative quantification of arginine methylation using nanoflow liquid chromatography coupled to electron transfer dissociation and orbitrap mass spectrometry. J Am Soc Mass Spectrom 20: 507-519.

Wang J, Saxe JP, Tanaka T, Chuma S, Lin H. 2009. Mili interacts with Tudor domain-containing protein 1 in regulating spermatogenesis. Curr Biol 19: 640-641.

Weinmann L, Hock J, Ivacevic T, Ohrt T, Mutze J, Schwille P, Kremmer E, Benes V, Urlaub H, Meister G. 2009. Importin 8 is a gene silencing factor that targets argonaute proteins to distinct mRNAs. Cell 136: 496-507.

Wilczynska A, Minshall N, Armisen J, Miska EA, Standart N. 2009. Two Piwi proteins, Xiwi and Xili, are expressed in the Xenopus female germline. RNA 15: 337-345.

Zgheib O, Pataky K, Brugger J, Halazonetis TD. 2009. An oligomerized 53BP1 tudor domain suffices for recognition of DNA double-strand breaks. Mol Cell Biol 29: 1050-1058. 


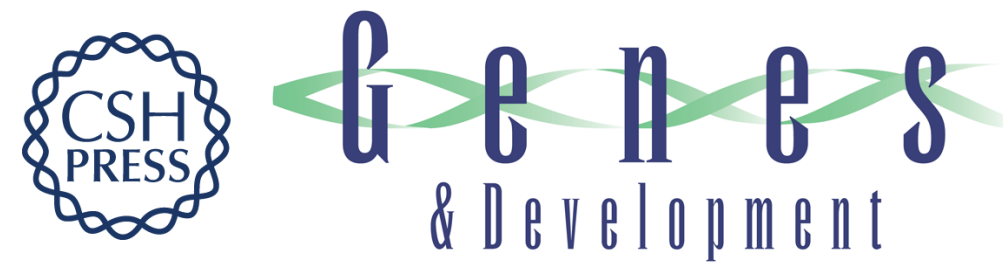

\section{Proteomic analysis of murine Piwi proteins reveals a role for arginine methylation in specifying interaction with Tudor family members}

Vasily V. Vagin, James Wohlschlegel, Jun Qu, et al.

Genes Dev. 2009, 23: originally published online July 7, 2009

Access the most recent version at doi:10.1101/gad.1814809

\section{Supplemental http://genesdev.cshlp.org/content/suppl/2009/07/09/gad.1814809.DC1 Material}

References This article cites 54 articles, 23 of which can be accessed free at: http://genesdev.cshlp.org/content/23/15/1749.full.html\#ref-list-1

License Freely available online through the Genes \& Development Open Access option. Email Alerting
Service $\begin{aligned} & \text { Receive free email alerts when new articles cite this article - sign up in the box at the top } \\ & \text { right corner of the article or click here. }\end{aligned}$

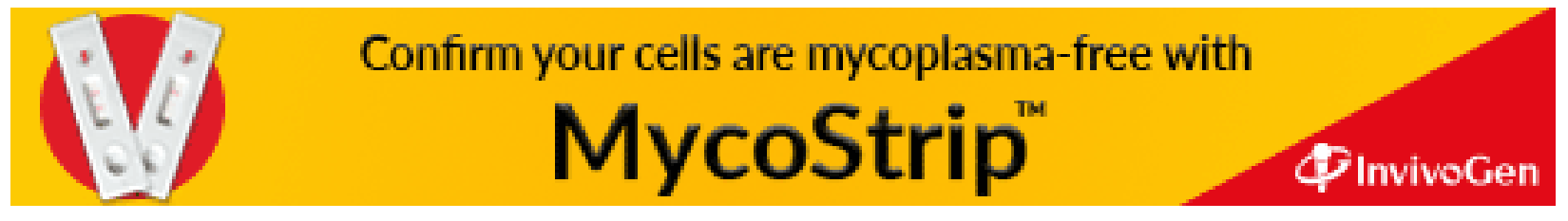

